SISSA $86 / 2005 / \mathrm{EP}$

\title{
Testing mass-varying neutrinos with reactor experiments
}

\author{
Thomas Schwetza*, Walter Winter ${ }^{\mathrm{b} \dagger}$

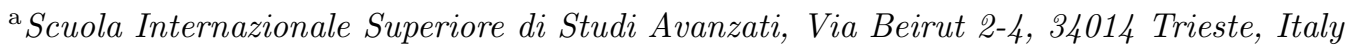 \\ ${ }^{\mathrm{b}}$ Institute for Advanced Study, School of Natural Sciences, Einstein Drive, Princeton, NJ 08540, USA
}

\begin{abstract}
We propose that reactor experiments could be used to constrain the environment dependence of neutrino mass and mixing parameters, which could be induced due to an acceleron coupling to matter fields. There are several short-baseline reactor experiment projects with different fractions of air and earth matter along the neutrino path. Moreover, the short baselines, in principle, allow the physical change of the material between source and detector. Hence, such experiments offer the possibility for a direct comparison of oscillations in air and matter. We demonstrate that for $\sin ^{2}\left(2 \theta_{13}\right) \gtrsim 0.04$, two reactor experiments (one air, one matter) with baselines of at least $1.5 \mathrm{~km}$ can constrain any oscillation effect which is different in air and matter at the level of a few per cent. Furthermore, we find that using the same experiment while physically moving the material between source and detector improves systematics.
\end{abstract}

PACS: $14.60 . \mathrm{Pq}$

Key words: Neutrino oscillations, Matter effects, long-baseline experiments

\section{Introduction}

The concept of mass-varying neutrinos (MVNs) has been introduced by imposing a relation between neutrinos and the dark energy of the Universe [1-4] through a scalar field, the acceleron. Including the possibility of acceleron couplings to matter fields implies that the neutrino oscillation parameters in vacuum and a medium could be very different [5], irrespective of the standard MSW effect [6,7]. MVNs can have substantial implications for neutrino phenomenology, for example in the sun [8-10] or for cosmology and astrophysics $[2,11,12]$, and they have been proposed as an explanation of the LSND anomaly $[5,13,14]$.

Given existing data, precision tests of massvarying effects are very difficult, since the different experiments are conducted under different conditions (different energies, baselines, matter distributions, etc.). Practically, all evidence for neutrino oscillations so far involves neutrino paths in (solar or earth) matter, whereas no direct information is available on oscillation parameters in vacuum or air. Moreover, the precise dependence of the neutrino masses on the matter density through the acceleron coupling is rather model dependent. The only plausible laboratory for a direct comparison between neutrino oscillations in different environments (e.g., matter and air) are short-baseline

*E-mail: schwetz@sissa.it

$\dagger$ E-mail: winter@ias.edu experiments. In this work, we consider the possibility to constrain MVNs with reactor experiments, which are planned to measure the mixing angle $\theta_{13}$ [15-17]. Such experiments are particularly suitable for this purpose, because one could think about building two very similar experiments with different material along the baseline, or even about moving the material along the baseline. In addition, reactor experiments suffer very little from correlations among the oscillation parameters, and new reactor experiments with identical near and far detectors will have an excellent sensitivity to $\sin ^{2}\left(2 \theta_{13}\right)$. Since the standard MSW effect is irrelevant for such short baselines, any deviation of the energy spectrum between matter and air can then be interpreted in terms of MVNs.

\section{General formalism}

For the reactor experiments under consideration, the ordinary matter potential is, to a first approximation, negligible because of the short baseline. The oscillation probability reads in the expansion up to second order in $\sin \left(2 \theta_{13}\right)$ and $\alpha \equiv$ $\Delta m_{21}^{2} / \Delta m_{31}^{2}$ (cf., e.g., Ref. [18])

$$
\begin{aligned}
1-P_{\bar{e} \bar{e}}= & \sin ^{2}\left(2 \theta_{13}\right) \sin ^{2} \Delta_{31}+ \\
& +\alpha^{2} \Delta_{31}^{2} \sin ^{2}\left(2 \theta_{12}\right),
\end{aligned}
$$

where $\Delta_{31} \equiv \Delta m_{31}^{2} L /(4 E)$. Thus, one can easily see that short-baseline reactor experiments have 
an excellent sensitivity to $\sin ^{2}\left(2 \theta_{13}\right)$. In particular, since $\alpha^{2} \simeq 10^{-3}$, one can read off from this equation that for large $\sin ^{2}\left(2 \theta_{13}\right) \gg 10^{-3}$ the second term is practically negligible, and we can use this formula in the two-flavor limit for analytical purposes.

Suppose that we have any non-standard effect on neutrino oscillations on the Hamiltonian level which is different in air and matter. In this case, we have the usual vacuum Hamiltonian $\mathcal{H}_{\text {vac }}$ in air, and some non-standard Hamiltonian $\mathcal{H}_{\text {mat }}=$ $\mathcal{H}_{\text {vac }}+\mathcal{H}_{\mathrm{ns}}$ in matter. In general, $\mathcal{H}_{\mathrm{ns}}$ is a Hermitian traceless ${ }^{3} n \times n$ matrix (for details, see e.g. Ref. [19]). The Hamiltonian $\mathcal{H}_{\text {mat }}$ can then be rediagonalized in order to obtain the effective mixing angles $\tilde{\theta}_{13}$ and $\Delta \tilde{m}_{31}^{2}$ in matter. In order to parameterize deviations between matter (tilde) and air (no tilde) parameters, we define

$$
\begin{aligned}
\delta_{\Delta} & =\Delta m_{31}^{2}-\Delta \tilde{m}_{31}^{2}, \\
\delta_{\theta} & =\theta_{13}-\tilde{\theta}_{13} .
\end{aligned}
$$

For MVNs one has $[8,10]$

$$
\mathcal{H}_{\text {mat }}=\frac{1}{2 E} U[\hat{m}-M(\rho)]^{\dagger}[\hat{m}-M(\rho)] U^{\dagger},
$$

where $\hat{m}=\operatorname{diag}\left(m_{i}\right)$ is the diagonal matrix of the neutrino masses and $U$ is the mixing matrix, both in the background dominated environment (e.g., air), and $M(\rho)$ is the mass matrix depending on the matter density $\rho$, which in general is non-diagonal in the basis used in Eq. (3). Hence, one finds that in the case of MVNs $\mathcal{H}_{\mathrm{vac}}$ and $\mathcal{H}_{\mathrm{ns}}$ have the same energy dependence, i.e., it is indeed the mass matrix which is modified. Note that according to Eq. (3), $\mathcal{H}_{\mathrm{ns}}$ also depends on the $m_{i}$.

In the following we will assume that the neutrinos propagate either through air or through matter with constant density. This allows a modelindependent test of MVNs, since only the density difference enters, and we need not to specify the detailed functional dependence of $M_{i j}(\rho)$. Furthermore, we assume that environment-dependent variations of $\Delta m_{21}^{2}$ and $\theta_{12}$ can be neglected, and the two parameters $\delta_{\Delta}$ and $\delta_{\theta}$ suffice to describe MVN effects for reactor experiments under consideration. This assumption can be justified by requiring the consistency of solar neutrino and KamLAND data, due to the much higher matter densities in the sun. The typical size of MVN parameters relevant for solar neutrinos $[8,10]$ is about one order of magnitude smaller than the sensitivity of

${ }^{3}$ If it is not traceless, it can be made traceless by the subtraction of a global phase. short-baseline reactor experiments, and in particular, if the bounds derived in Ref. [10] are applied, MVN effects on $\Delta m_{21}^{2}$ and $\theta_{12}$ can be neglected (see also Sec. 17). We stress that reactor experiments test different MVN parameters than solar neutrino experiments.

Let us add, that for non-standard matter effects apart from MVNs (see Ref. [20] for a review) the energy dependence of $\mathcal{H}_{\mathrm{ns}}$ may be different than the one of $\mathcal{H}_{\mathrm{vac}}$. For instance, there may be a relative factor $1 /(2 E)$ between $\mathcal{H}_{\text {vac }}$ and $\mathcal{H}_{\text {ns }}$ leading to energy-dependent matter parameters similar to the standard MSW matter effect (cf., Ref. [19]). In this case, the assumption of an energy-independent effect can still be used as an approximation for short-baseline reactor experiments (by using the peak energy of the spectrum) as long as the neutrino energy is far away from the MSW resonance energy. This requirement is only violated if the non-standard effects are more than two orders of magnitude larger than the standard MSW matter effect.

\section{Reactor experiment geometries}

In principle, we expect to obtain the best MVN sensitivities by the comparison of a matter to an air experiment. As we will see, using reactor experiments for the test of MVNs is mainly a matter of geometry. Some experiments have their baseline in air (such as Double Chooz; see, e.g., Refs. [13,14]), and others have it mostly in matter or mixed between air and matter sections. In addition, moving detectors have been discussed. Currently, three major principle schemes of reactor experiment layouts, which can be found in Fig. 1 are:

Type (a) Baseline mostly in air. Example: Double Chooz [21].

Type (b) Baseline entirely in matter because of flat terrain. Example: Braidwood [22], KASKA [23].

Type (c) Baseline mostly in matter because of hills. Examples: Daya Bay [24], Angra [25].

One could now think about three different conceptual cases to test MVN effects. First, one could use existing (future) experiments with baselines in air and matter and combine them, such as Double Chooz or a larger reactor experiment in air combined with another reactor experiment in matter, or the long-baseline beam experiments T2K or $\mathrm{NO} \nu \mathrm{A}$. Second, one could modify the existing 


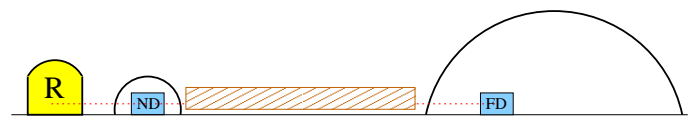

(a) Mostly air

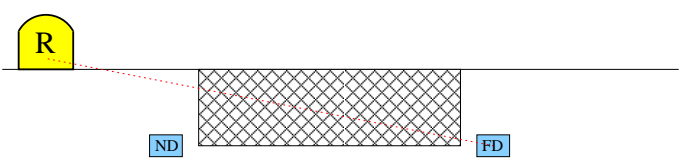

(b) Flat terrain, underground

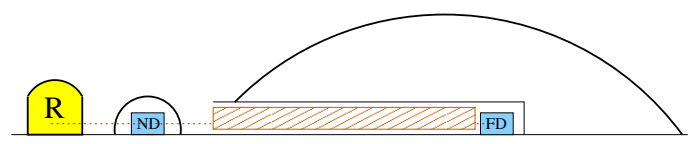

(c) Hills, mostly underground

Figure 1. Schematic illustration of different types of reactor experiment terrain layouts and potential modifications for the test of non-standard matter effects (not to scale). The filled boxes in (a) and (c) refer to additions of material in the far detector's line of sight, and the box in (b) refers to removing material in the far detector's line of sight after the primary operation period.

experiments. In phase I, the experiment runs unmodified, and in a subsequent phase II the material in the line of sight of the far detector is changed. Examples of these modifications can be found in Fig. 11 For type (a), the far detector's line of sight could be covered with Earth or a rock wall. For type (b), a hole in the ground would lead to air propagation in the far detector's line of sight. And for type (c), the access tunnel to the far detector could a priori be built in the line of sight of far detector which is then covered with Earth or rock wall in phase II. Alternatively, one could drill an additional tunnel. As the last conceptual case, one could use a vertically or horizontally moving detector and the geometry of the site to change the material between source and detector.

\section{Analysis methods}

For the experiment simulation, we use a version of the GLoBES software [26] with some modifications to simulate non-standard physics $[27,28]$. For the experiments, we mainly use the simulations of the Reactor-II and Double Chooz [21] setups from Refs. $[16,29]$. Reactor-II is an abstract background-free reactor experiment with identical near and far detectors, a baseline $L_{\mathrm{far}}=1.7 \mathrm{~km}$, and an integrated luminosity of $8000 \mathrm{tGWyr}$. There are now many proposals for an experiment similar to this setup ( $c f$. ., Ref. [17], and references therein). For Double Chooz, we use a backgroundfree simulation with an integrated 60000 unoscillated events, and a baseline $L_{\mathrm{far}}=1.05 \mathrm{~km}$, which should be a very good approximation for the actual experiment. In addition, we will show some results in combination with $\mathrm{NO} \nu \mathrm{A}$ instead of a reactor experiment in matter, where we use the simulation based upon Refs. [30,31] adjusted to the proposal parameters [32] ( $L=810 \mathrm{~km}, 12 \mathrm{~km}$ off-axis, $30 \mathrm{kt}$ TASD) with a running time of five years in the neutrino mode.

We simulate the data for a given set of "true" oscillation parameters [33-36] $\Delta m_{31}^{2}=2.2 \cdot 10^{-3} \mathrm{eV}^{2}$, $\sin ^{2} 2 \theta_{23}=1, \Delta m_{21}^{2}=8.2 \cdot 10^{-5} \mathrm{eV}^{2}, \sin ^{2} 2 \theta_{12}=$ 0.83 , and $\sin ^{2}\left(2 \theta_{13}\right)=0.1$ somewhat below the $\mathrm{CHOOZ}$ bound [37], $\delta_{\mathrm{CP}}=0$ (relevant for beams only), and a normal hierarchy, and we assume no MVN effect in the "data", i.e., true $\delta_{\Delta}=\delta_{\theta}=0$. Then we perform a fit to these simulated data, and we project the fit manifold onto the $\delta_{\Delta}-\delta_{\theta}$ plane by marginalization of the standard oscillation parameters. For this process, we impose external precisions of $5 \%$ for each $\Delta m_{21}^{2}$ and $\theta_{12}$ [34], which should be realistic estimates for the analysis time. We do not include the wrong hierarchy solution, because there is a good chance that the mass hierarchy will be measured on the timescale we are discussing (see, e.g., Refs. $[29,32]){ }^{4}$

\section{Systematics treatment}

Before we present our results, we need to discuss the reactor experiment systematics in greater detail. We use a treatment similar to Ref. [16] for a reactor experiment with two identical detectors, where the main systematics impact was identified as an effective normalization error of about $0.8 \%$. Here we consider two different cases:

1. One experiment with two phases: Phase I mainly in matter, phase II mainly in air. The detectors are not only identical, but also physically the same.

2. Two different experiments, one mainly in air,

\footnotetext{
${ }^{4}$ Because reactor experiments are insensitive to the mass hierarchy, the results do, in principle, not depend on the mass hierarchy. However, the sign of the $\delta_{\Delta}$-effects could change for a different mass hierarchy, because $\delta_{\Delta}$ may come from a specific shift of one of the mass eigenstates.
} 
the other mainly in matter. The detectors are identical, but not physically the same.

We write the $\chi^{2}$ for both cases as

$$
\begin{aligned}
& \chi^{2}=\sum_{x=m, a} {\left[\sum_{i} \frac{\left[\left(1+b+b_{x}\right) N_{i}^{x}-O_{i}\right]^{2}}{O_{i}}\right.} \\
&\left.+\left(\frac{b_{x}}{\sigma_{u c}}\right)^{2}\right]+\left(\frac{b}{\sigma_{c}}\right)^{2},
\end{aligned}
$$

where $N_{i}\left(O_{i}\right)$ are the oscillated (unoscillated) event numbers in energy bin $i$, the index $x$ labels the data taken in air $(a)$ and in matter $(m)$, and one has to minimize Eq. (44) with respect to $b, b_{a}$, and $b_{m}$. The error $\sigma_{u c}$ corresponds to an uncorrelated normalization error between the air and matter data, whereas $\sigma_{c}$ is fully correlated. The interpretation of $\sigma_{u c}$ and $\sigma_{c}$ depends on the setup and will be discussed below.

Let us do some analytical estimations. First, we will see that the systematics mainly affects the $\sin ^{2}\left(2 \theta_{13}\right)$ measurement, which means that we will be only interested in the $\sin ^{2}\left(2 \theta_{13}\right)$ measurement in this section. Second, for simplicity, we consider only a rate measurement. Then the oscillated event rates can be written as $N^{x}=\left(1-S_{x} f_{x}\right) \mathcal{N}$, where $S_{x} \equiv \sin ^{2}\left(2 \theta_{13}^{x}\right)$,

$$
f_{x} \equiv\left\langle\sin ^{2} \frac{\Delta m_{x}^{2} L}{4 E}\right\rangle=\mathcal{O}(1),
$$

and $\mathcal{N} \equiv O$ is the total number of events in one experiment. Using $S_{x}, b, b_{x} \ll 1$, the $\chi^{2}$ can be linearized. Since we are interested in the absolute error $\sigma_{S}$ on $S_{a}-S_{m}$, we then minimize the linearized Eq. (44) with respect to $b, b_{a}$, and $b_{m}$, and compute $\sigma_{S}$ taking into account the correlation matrix between $S_{a}$ and $S_{m}$ in order to obtain

$\sigma_{S}^{2} \simeq\left(\sigma_{u c}^{2}+\frac{1}{\mathcal{N}}\right)\left(\frac{1}{f_{a}^{2}}+\frac{1}{f_{m}^{2}}\right)+\sigma_{c}^{2}\left(\frac{1}{f_{a}}-\frac{1}{f_{m}}\right)^{2}$.

For case 2 (two separate experiments), $\sigma_{u c}$ corresponds to the effective normalization error of each individual experiment, and the second term of Eq. (6) can be neglected because it is usually small compared to the first one. As one may expect, in the (poor) statistics dominated regime $1 / \sqrt{\mathcal{N}} \gg \sigma_{u c}$, the error scales as $1 / \sqrt{\mathcal{N}}$, whereas for large event numbers $1 / \sqrt{\mathcal{N}} \ll \sigma_{u c}$ the error is limited by the systematical uncertainty $\sigma_{u c}$ (cf., also Refs. [38-40] for similar considerations).
For Reactor-II, we have $\mathcal{N} \sim 630000$ unoscillated events and $\sigma_{u c} \simeq 0.8 \%$, which means that $1 / \sqrt{\mathcal{N}} \sim 0.001 \ll \sigma_{u c}$, and we are in the systematics dominated regime.

For case 1 (one experiment), however, $\sigma_{u c}$ is expected to be very small, since it only contains the time-dependent evolution of the systematics which is different for the near and far detectors. We only have to worry about values larger than about $1 / \sqrt{\mathcal{N}}$, which is $\simeq 0.1 \%$ for a Reactor-II setup, and we neglect it for the moment. In the statistics dominated regime $1 / \sqrt{\mathcal{N}} \gtrsim \sigma_{c}$, one can neglect the second term in Eq. (6) and one recovers the same limiting expression as for the two-experiment setup. For large $\mathcal{N}$ (and neglecting $\sigma_{u c}$ ), the second term limits the accuracy. The $1 / \sqrt{\mathcal{N}}$ scaling is cut off when the condition

$$
\sigma_{c}^{2} \frac{\left(f_{a}-f_{m}\right)^{2}}{f_{a}^{2} f_{m}^{2}} \ll \frac{1}{\mathcal{N}}
$$

is violated. Note that $\left(f_{a}-f_{m}\right)^{2}$ is a small number: Using Eq. (5) one finds $f_{a}-f_{m} \simeq$ $\epsilon\left\langle\sin \left(\Delta m_{a}^{2} L / 2 E\right)\right\rangle$, where $\epsilon \sim \delta_{\Delta} / \Delta m_{a}^{2} \lesssim 0.1$. Furthermore, also the second factor is small, since the fact that the experiments work at the oscillation maximum implies $\Delta m_{a}^{2} L / 2 E \simeq \pi$. Using the numbers for Reactor-II, it is straight forward to estimate that the condition in Eq. (7) is fulfilled, and hence, it is justified to neglect $\sigma_{c}$ for a oneexperiment setup. We conclude that the case 1 stays up to very high event numbers in the statistics dominated regime, and we simulate it in the next section by the most optimistic assumption of a systematics-free measurement.

\section{Phenomenological results}

Let us first discuss the case of using experiments proposed for measuring the standard oscillation parameters. We show in Fig. 2] left, the combined sensitivity of Double Chooz (air) and the reactor experiment Reactor-II (matter). Obviously, there is a strong correlation between $\delta_{\Delta}$ and $\delta_{\theta}$, which one can understand as follows: The experiment Reactor-II measures the matter parameters because of the much better statistics. For $\delta_{\Delta}>0$, the $\Delta m_{31}^{2}$ in air can be measured by Double Chooz by the spectral information. However, for $\delta_{\Delta}<0$, the oscillation phase becomes $\Delta_{31} \ll 1$, and the oscillation probability in Eq. (11) can be expanded as $1-P_{\bar{e} \bar{e}} \simeq \sin ^{2}\left(2 \theta_{13}\right) \Delta_{31}^{2}+\ldots$. This implies that a smaller $\delta_{\Delta}$ (changing $\Delta m_{31}^{2}$ ) can be directly compensated by a larger $\delta_{\theta}$ (changing $\theta_{13}$ ), which can be clearly seen in Fig. 2] left. The final precision 

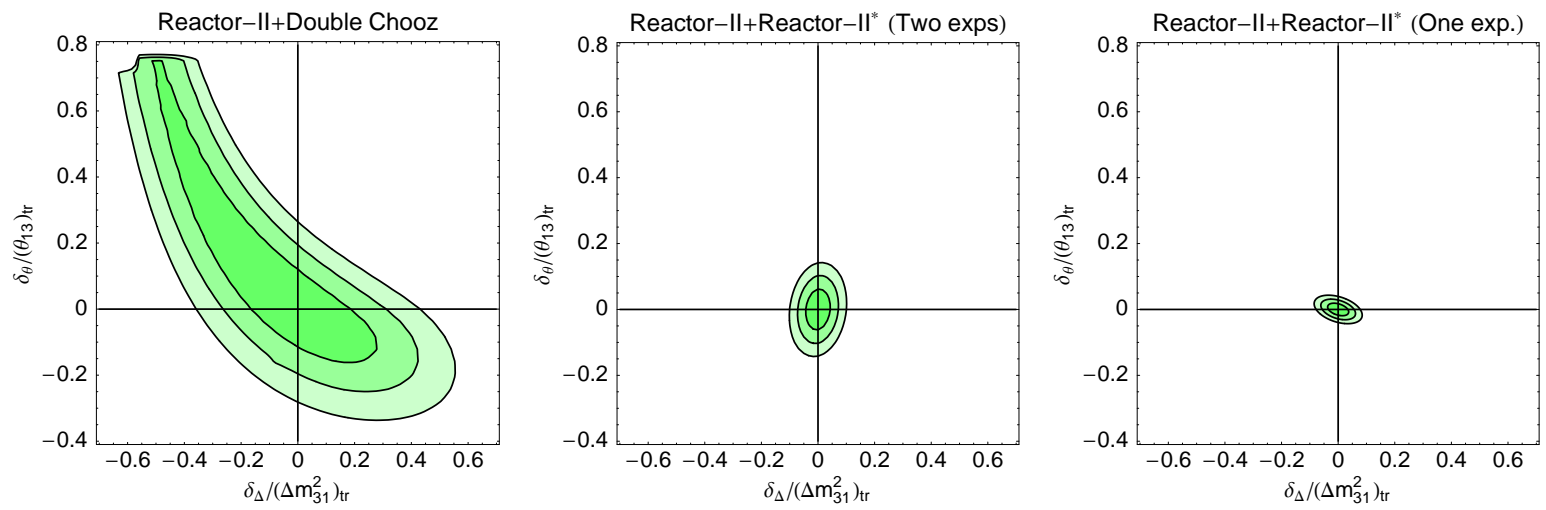

Figure 2. The combined sensitivities to $\delta_{\Delta}$ and $\delta_{\theta}$ (as relative changes between air and matter) for $\sin ^{2}\left(2 \theta_{13}\right)_{\mathrm{tr}}=0.1$ at the $1 \sigma, 2 \sigma$, and $3 \sigma$ CL (2 d.o.f) for different experiments. The left panel refers to Double Chooz combined with a large reactor experiment Reactor-II in matter, the middle panel refers to two different experiments Reactor-II (matter) + Reactor-II* (100 m matter, $1500 \mathrm{~m}$ air, $100 \mathrm{~m}$ matter), and the right panel refers to the optimal case with the same experiment Reactor-II (matter, phase I) + Reactor-II* (phase II), i.e., actually the material is moved.

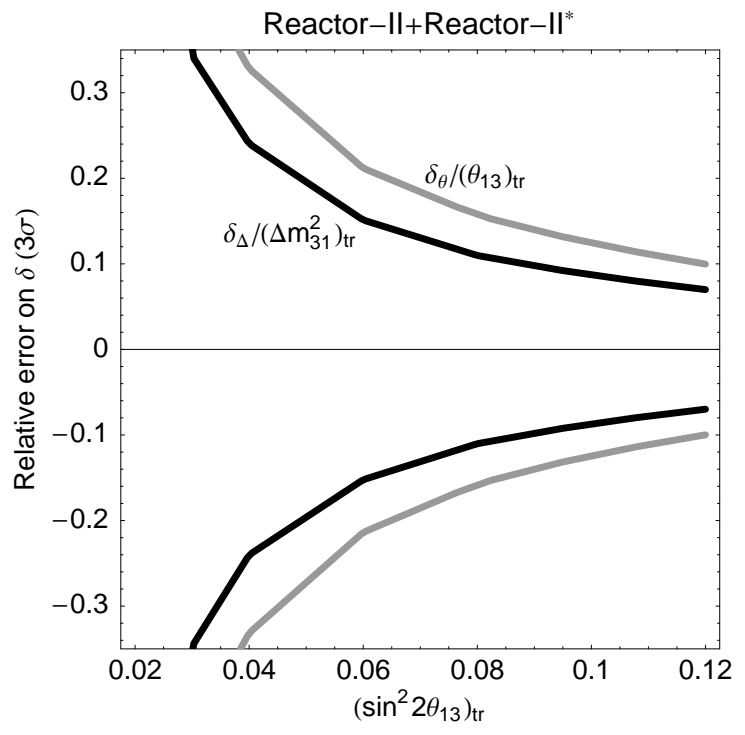

Figure 3. The sensitivities to $\delta_{\Delta}$ and $\delta_{\theta}$ (as relative changes between air and matter) at the $3 \sigma$ CL (1 d.o.f) as a function of the true $\sin ^{2}\left(2 \theta_{13}\right)$ for two different experiments Reactor-II (matter) + Reactor-II* (100 m matter, $1500 \mathrm{~m}$ air, $100 \mathrm{~m}$ matter).

for $\delta_{\Delta}$ and $\delta_{\theta}$ is only comparable to the order of $\Delta m_{31}^{2}$ and $\sin ^{2}\left(2 \theta_{13}\right)$ themselves.

As a next step, one could think about combining two very similar reactor experiments, one in air and one almost in matter. We show in Fig. 2. middle, the results for this setup for two experiments with $L=1.7 \mathrm{~km}$. Since both experiments practically have the same spectrum, the precision on $\delta_{\Delta}$ and $\delta_{\theta}$ is extremely improved (below $10 \%$ at $3 \sigma, 1$ d.o.f.). The correlation between $\delta_{\Delta}$ and $\delta_{\theta}$ has almost completely disappeared, because of the long enough baseline. We find that there is no strong baseline dependence for $1.5 \mathrm{~km} \lesssim L \lesssim$ $3.5 \mathrm{~km}$, as can be seen from the lower part of Table 11 In Fig. 3 we show the dependence of the sensitivity on $\sin ^{2}\left(2 \theta_{13}\right)$. One observes that for $\sin ^{2}\left(2 \theta_{13}\right) \gtrsim 0.04$, the difference of the parameters in air and matter can be constrained with a precision of better than about $30 \%$ at the $3 \sigma$ CL. ${ }^{5}$ Similar results can be obtained for the combination of an air Double Chooz or Reactor-II experiment and a superbeam, such as $\mathrm{T} 2 \mathrm{~K}$ [41] or $\mathrm{NO} \nu \mathrm{A}$ [32] ( $c f$., Table (1). In this case the sensitivity to $\delta_{\theta}$ is somewhat degraded because of additional correlations with $\delta_{\mathrm{CP}}$ and $\theta_{23}$ in the beam experiment.

Finally, we show in Fig. 2 right, the most optimistic results for the same experiment, i.e., using Reactor-II when $1.5 \mathrm{~km}$ of the matter is removed after the phase I run. For both phases we assume a running time of 5 years. As discussed in the previous section, we simulate this situation by setting all systematical errors to zero, which leads to a further improvement of the sensitivity to $\delta_{\theta}$. As highlighted in Table 11 such a setup allows a test of MVNs at the level of a few per cent at $3 \sigma$ CL.

\footnotetext{
${ }^{5}$ Note that due to the small values of $\theta_{13} \sim 0.1$ a relative precision of $30 \%$ implies the impressive accuracy of $\sim 0.03$ for $\delta_{\theta}$.
} 


\begin{tabular}{lrr}
\hline Experiment combination & $\delta_{\Delta} /\left(\Delta m_{31}^{2}\right)_{\mathrm{tr}}$ & $\delta_{\theta} /\left(\theta_{13}\right)_{\mathrm{tr}}$ \\
\hline Double Chooz (air) + Reactor-II (matter) & $0.63(0.29)$ & $0.77(0.29)$ \\
Double Chooz (air) + NO $\nu$ A (matter) & $0.75(0.30)$ & $>1(0.34)$ \\
Reactor-II (matter) + Reactor-II* & $0.09(0.03)$ & $0.12(0.04)$ \\
Reactor-II (matter), transformed into Reactor-II* & $\mathbf{0 . 0 7 ( 0 . 0 2 )}$ & $\mathbf{0 . 0 4}(\mathbf{0 . 0 1})$ \\
Reactor-II (air) + NO $\nu$ A (matter) & $0.08(0.03)$ & $0.37(0.23)$ \\
\hline Reactor-II + Reactor-II*, $L=1.0 \mathrm{~km}$ & $0.33(0.10)$ & $0.20(0.05)$ \\
Reactor-II + Reactor-II*,$L=2.0 \mathrm{~km}$ & $0.08(0.02)$ & $0.13(0.04)$ \\
Reactor-II + Reactor-II*,$L=3.0 \mathrm{~km}$ & $0.07(0.01)$ & $0.13(0.04)$ \\
\hline
\end{tabular}

Table 1

Relative sensitivities to $\delta_{\Delta}$ and $\delta_{\theta}$ at the $3 \sigma(1 \sigma)$ CL (1 d.o.f.) for different experimental setups and $\sin ^{2}\left(2 \theta_{13}\right)_{\mathrm{tr}}=0.1$. If the upper and lower bounds are different, the maximum value is given. The definition of Reactor-II* is $100 \mathrm{~m}$ matter, $1500 \mathrm{~m}$ air, $100 \mathrm{~m}$ matter, whereas Reactor-II is in matter unless stated otherwise. The standard value for the baseline of Reactor-II is $L=1.7 \mathrm{~km}$ unless stated otherwise.

\section{Implications for mass-varying neu- trino models}

In the preceding section, we have demonstrated that reactor experiments could provide powerful tests of any neutrino oscillation model which is different in air and matter in the $\Delta m_{31}^{2}-\sin ^{2}\left(2 \theta_{13}\right)$ sector. Now we discuss some mass-varying neutrino model-dependent aspects.

Let us first estimate the sensitivity to the elements $M_{13}, M_{33}$ of the environment-dependent mass matrix in Eq. (3). For simplicity we take $M_{11}=0$. Assuming an accuracy $\epsilon \sim \delta_{\Delta} / \Delta m_{31}^{2} \sim$ $\delta_{\theta} / \theta_{13}$, a rough estimation gives a sensitivity of $M_{13}, M_{33} \lesssim \epsilon \sqrt{\Delta m_{31}^{2}}$. Now one can consider for example the following model for the environment-dependent mass matrix $[8,10]: M_{i j}=$ $\lambda_{i j}^{\nu} \lambda^{f} n_{f} / m_{S}^{2}$, where $f$ denotes a fermion in the medium, $\lambda_{i j}^{\nu}\left(\lambda^{f}\right)$ are the Yukawa couplings of neutrinos (the fermion $f$ ) to the acceleron, $n_{f}$ is the number density of $f$, and $m_{S}$ is the acceleron mass. If we consider only the coupling to electrons, we have $n_{e} \simeq 6.4 \cdot 10^{9} \mathrm{eV}^{3}$ in earth matter (for $\rho=2.8 \mathrm{~g} / \mathrm{cm}^{3}$ ), and we find the following order of magnitude for the sensitivity of reactor experiments to the model parameters:

$$
\left|\lambda^{\nu} \lambda^{e}\right|\left(\frac{10^{-7} \mathrm{eV}}{m_{S}}\right)^{2} \lesssim 2 \cdot 10^{-27}\left(\frac{\epsilon}{0.03}\right)
$$

This number has to be compared to the value $3 \cdot 10^{-28}$ obtained in Ref. [10] for the sensitivity of solar neutrino data to MVN parameters in the 1-2 sector. Naively one expects that solar neutrino experiments are much more sensitive to MVN effects than reactor experiments, since there is roughly a factor of 100 between the densities in the solar core and earth matter, and there is another factor $\sqrt{\Delta m_{31}^{2} / \Delta m_{21}^{2}} \sim 5$, which characterizes the mass scale sensitivity of the experiments. However, these factors are partially compensated by the high precision of reactor experiments, $\epsilon \sim 0.03$.

In principle solar+KamLAND neutrino data have some sensitivity to $\theta_{13}[33-36]$. The above argument on the factor 100 between the densities in the sun and the earth suggests that solar data should also be able to provide some information on MVN effects for $\theta_{13}$. Such a constraint requires model-dependent assumptions about the density dependence, since one has to relate the environment-dependent values of $\theta_{13}$ inside the sun, in earth matter, and in air. Depending on such model assumptions, one could have constraints on $\delta_{\theta}$ from solar neutrino data, which could change the interpretation of our results: For $\delta_{\theta}$ fixed to 0 , already planned experiments in matter in combination with Double Chooz will provide very good results for $\delta_{\Delta}$ ( $c f$., left panel of Fig. 2).

A very particular mass-varying neutrino model has been considered in Ref. [14] to explain the LSND experiment and a potential null result in MiniBOONE by the combination of mass-varying neutrinos and one additional sterile neutrino mixing with the active ones in matter. Since this model predicts no oscillations in air for shortbaseline reactor experiments, a signal for $\sin ^{2}\left(2 \theta_{13}\right)$ in air (for example in Double Chooz) would be a strong rejection of this model, i.e., $\delta_{\Delta}$ has to be tested only of the order of one. Furthermore, our setups can test any additional sterile neutrinos with mixings different in air and matter $[11,14]$ as follows: If the fast oscillation frequency associated with the sterile neutrinos $\Delta M^{2}$ leads to oscillation effects already at the near detector site (such as for $\Delta M^{2} \gtrsim 1 \mathrm{eV}^{2}$ ), then both the near and far detectors will observe a reduced overall flux. This can neither be faked by $\sin ^{2}\left(2 \theta_{13}\right)$ nor $\Delta m_{31}^{2}$ being 
different in matter. Thus, a sterile neutrino contribution in matter can be measured on the level the reactor flux is known, typically some per cent, see e.g., Ref. [42].

\section{Summary and conclusions}

We have considered several reactor experiment setups with baselines in air and matter to constrain any non-standard contribution to the Hamiltonian which is different in air and matter. In particular, we find that new reactor experiments with near and far detectors could provide stringent bounds for mass-varying neutrino models, which lead to environment dependent effects in the 1-3 sector $\left(\Delta m_{31}^{2}\right.$ or $\left.\sin ^{2}\left(2 \theta_{13}\right)\right)$.

Non-trivial constraints can be obtained already from Double Chooz (mainly air) combined with a matter experiment, although in this case correlations between the parameters limit the sensitivity. Best results, however, are obtained if two identical setups with baselines of at least $1.5 \mathrm{~km}$ with substantially different material between the near and far detectors are compared. For $\sin ^{2}\left(2 \theta_{13}\right) \gtrsim 0.04$, deviations of oscillation parameters in matter and air can be constrained at the level of few per cent. Using the same experiment and physically moving the matter between the two detectors after the initial operation period decreases the impact of systematical errors. In this case, the relevant systematics issue is the uncorrelated time-dependent change between the near and far detectors, which should be extremely small for practical purposes. We conclude that new reactor experiments could be excellent candidates for the test of mass-varying neutrinos provided that $\sin ^{2}\left(2 \theta_{13}\right)$ is not to small.

\section{Acknowledgments}

We thank P. Huber, M. Maltoni and R. Zukanovich for illuminating discussions. Furthermore, we would like to thank J. Anjos, M. Goodman, and Y. Wang for useful information. WW would like to acknowledge support from the W. M. Keck Foundation and NSF grant PHY-0503584. TS is supported by a "Marie Curie Intra-European Fellowship within the 6th European Community Framework Program."

\section{REFERENCES}

1. P. Q. Hung (2000), hep-ph/0010126

2. P. Gu, X. Wang, and X. Zhang, Phys. Rev. D68, 087301 (2003), hep-ph/0307148
3. R. Fardon, A. E. Nelson, and N. Weiner, JCAP 0410, 005 (2004), astro-ph/0309800

4. R. D. Peccei, Phys. Rev. D71, 023527 (2005), hep-ph/0411137.

5. D. B. Kaplan, A. E. Nelson, and N. Weiner, Phys. Rev. Lett. 93, 091801 (2004), hep-ph/0401099.

6. L. Wolfenstein, Phys. Rev. D17, 2369 (1978).

7. S. P. Mikheev and A. Y. Smirnov, Sov. J. Nucl. Phys. 42, 913 (1985).

8. V. Barger, P. Huber, and D. Marfatia (2005), hep-ph/0502196

9. M. Cirelli, M. C. Gonzalez-Garcia, and C. Pena-Garay, Nucl. Phys. B719, 219 (2005), hep-ph/0503028

10. M. C. Gonzalez-Garcia, P. C. de Holanda, and R. Z. Funchal (2005), hep-ph/0511093

11. N. Weiner and K. Zurek (2005), hep-ph/0509201.

12. P. Q. Hung and H. Pas, Mod. Phys. Lett. A20, 1209 (2005), astro-ph/0311131.

13. K. M. Zurek, JHEP 10, 058 (2004), hep-ph/0405141.

14. V. Barger, D. Marfatia, and K. Whisnant (2005), hep-ph/0509163

15. H. Minakata, H. Sugiyama, O. Yasuda, K. Inoue, and F. Suekane, Phys. Rev. D68, 033017 (2003), hep-ph/0211111

16. P. Huber, M. Lindner, T. Schwetz, and W. Winter, Nucl. Phys. B665, 487 (2003), hep-ph/0303232

17. K. Anderson et al. (2004), hep-ex/0402041.

18. E. K. Akhmedov, R. Johansson, M. Lindner, T. Ohlsson, and T. Schwetz, JHEP 04, 078 (2004), hep-ph/0402175

19. M. Blennow, T. Ohlsson, and W. Winter (2005), hep-ph/0508175

20. J. W. F. Valle, Prog. Part. Nucl. Phys. 26, 91 (1991).

21. F. Ardellier et al. (2004), hep-ex/0405032.

22. T. Bolton, Nucl. Phys. Proc. Suppl. 149, 166 (2005).

23. M. Kuze (KASKA), Nucl. Phys. Proc. Suppl. 149, 160 (2005), hep-ex/0502002

24. J. Cao (2005), hep-ex/0509041

25. J. Anjos et al. (2005), talk given at $\mathrm{Nu}-$ Fact 2005, Frascatti, Italy, available at http://www.e-science.unicamp.br/nangra/

26. P. Huber, M. Lindner, and W. Winter, Comput. Phys. Commun. 167, 195 (2005), http://www.ph.tum.de/ globes hep-ph/0407333

27. M. Blennow, T. Ohlsson, and W. Winter, JHEP 06, 049 (2005), hep-ph/0502147. 
28. P. Huber, J. Kopp, M. Lindner, M. Rolinec, and W. Winter GLoBES new feature release, in preparation.

29. P. Huber, M. Lindner, M. Rolinec, T. Schwetz, and W. Winter, Phys. Rev. D70, 073014 (2004), hep-ph/0403068

30. P. Huber, M. Lindner, and W. Winter, Nucl. Phys. B645, 3 (2002), hep-ph/0204352

31. P. Huber, M. Lindner, and W. Winter, Nucl. Phys. B654, 3 (2003), hep-ph/0211300

32. D. S. Ayres et al. (NOvA) (2004), hep-ex/0503053

33. G. L. Fogli, E. Lisi, A. Marrone, and A. Palazzo (2005), hep-ph/0506083

34. J. N. Bahcall, M. C. Gonzalez-Garcia, and C. Peña Garay, JHEP 08, 016 (2004), hep-ph/0406294

35. A. Bandyopadhyay, S. Choubey, S. Goswami, S. T. Petcov, and D. P. Roy, Phys. Lett. B608, 115 (2005), hep-ph/0406328

36. M. Maltoni, T. Schwetz, M. A. Tortola, and J. W. F. Valle, New J. Phys. 6, 122 (2004), hep-ph/0405172

37. M. Apollonio et al. (CHOOZ), Eur. Phys. J. C27, 331 (2003), hep-ex/0301017.

38. H. Sugiyama, O. Yasuda, F. Suekane, and G. A. Horton-Smith (2004), hep-ph/0409109

39. H. Sugiyama and O. Yasuda (2005), hep-ph/0508090

40. P. Huber, M. Lindner, and T. Schwetz, JHEP 02, 029 (2005), hep-ph/0411166

41. Y. Itow et al., Nucl. Phys. Proc. Suppl. 111, 146 (2001), hep-ex/0106019

42. P. Huber and T. Schwetz, Phys. Rev. D70, 053011 (2004), hep-ph/0407026 